\title{
Calidad de vida familiar y TDAH infantil. Perspectiva multidisciplinar desde la educación física y el trabajo social
}

\author{
Quality of family life and childhood ADHD. Multidisciplinary perspective \\ from physical education and social work
}

\author{
Diana Karely Quintero-Olivas, Ena Monserrat Romero Pérez \& \\ José Aldo Hernández-Murúa
}

\begin{abstract}
Quintero-Olivas, D., Romero, E. M., \& Hernández-Murúa, J. A. (2021). Calidad de vida familiar y TDAH infantil. Perspectiva multidisciplinar desde la educación física y el trabajo social. Revista Ciencias de la Actividad Física UCM, N²2(1), enero-junio, 1-17. DOI: http://doi.org/10.29035/rcaf.22.1.1
\end{abstract}

\section{RESUMEN}

La familia ha sido considerada el contexto social que más influencia ejerce para la formación y desarrollo de cualquier individuo. El objetivo de este trabajo es destacar la importancia de la calidad de vida de las familias que experimentan la presencia de un integrante con Trastorno por Déficit de Atención e Hiperactividad (TDAH) y cómo, desde las áreas profesionales de educación física y trabajo social, se puede plantear una propuesta para su tratamiento integral. Para ello, se revisan diversos estudios publicados en los últimos años, analizando las diferentes formas en las que han abordado la calidad de vida en el contexto familiar. Se espera que el análisis de estos aportes incite en la innovación y promoción de nuevas formas de abordar la calidad de vida familiar en infantes con TDAH.

Palabras clave: TDAH, Educación Física, Trabajo Social, Calidad de Vida Familiar.

\begin{abstract}
Family has been identified as the most influential social context for the formation and development of any individual. The objective of this work is to highlight the importance of the quality of life of families that experience the presence of a member with attention deficit hyperactivity disorder (ADHD) and through the professional areas of physical education and social work, an approach for its comprehensive treatment is given. To do this, several studies published in recent years were reviewed, analyzing the different ways in which they have addressed the quality of life in the family context. The analysis of these contributions is expected to influence the innovation and promotion of new ways by addressing the quality of family life in infants with ADHD.
\end{abstract}

Key words: ADHD, Physical Education, Social Work, Quality of Family Life.

\footnotetext{
* Maestría en Ciencias. Facultad de Trabajo Social, Universidad Autónoma de Sinaloa, Culiacán, México. ID: https://orcid.org/0000-0001-7804-0756 dianaquintero1994@gmail.com

** Doctora en Ciencias de la Actividad Física y Deporte Universidad de Sonora, Hermosillo, México.

ID: https://orcid.org/0000-0003-2965-9979 ena-romero@unison.mx

*** Doctor en Ciencias. Facultad de Educación Física y Deporte, Universidad Autónoma de Sinaloa, Culiacán, México. ID: https://orcid.org/0000-0002-9277-5799 aldohdez80@hotmail.com
} 
Quintero-Olivas, D., Romero, E. M., \& Hernández-Murúa, J. A. (2021). Calidad de vida familiar y TDAH infantil. Perspectiva multidisciplinar desde la educación física y el trabajo social. Revista Ciencias de la Actividad Física UCM, № 22(1), enero-junio, 1-17. DOI: http://doi.org/10.29035/rcaf.22.1.1

\section{INTRODUCCIÓN}

El Trastorno por Déficit de Atención e Hiperactividad (TDAH) se caracteriza por ser un trastorno multifactorial, el cual no puede explicarse solo por una causa, pues están involucradas vulnerabilidades biológicas, las cuales al mismo tiempo están expuestas a una variedad de factores ambientales que perpetúan el trastorno. Éste se distingue por una serie de comportamientos que tienen repercusiones en el funcionamiento cognitivo, educacional, social y familiar de la persona que lo padece (Quintero \& Castaños, 2014).

La Secretaria de Salud (SSA) (2002) define que este trastorno es una afección neuropsiquiátrica, se caracteriza por la falta de atención, la hiperactividad y la impulsividad; puede presentarse con o sin hiperactividad en diversos casos. Los niños que lo padecen presentan problemas de conducta, estados de ánimo inestables, ansiedad y problemas de aprendizaje, lo cual repercute de manera importante en el ámbito familiar, social y escolar.

EI TDAH ha cobrado gran relevancia debido a que éste no solo implica un problema de salud individual; su presencia puede generar afectaciones en la familia o viceversa. En el contexto familiar, la presencia de un integrante con este trastorno puede perturbar su funcionamiento, como consecuencia, todo el grupo pasa por momentos difíciles por no saber cómo enfrentar las situaciones que experimentan y, al final, cualquier intento de tratamiento médico o conductual para mejorar los síntomas del TDAH en los niños que lo padecen, puede verse trastornado e ineficaz debido a la alteración del funcionamiento familiar, y a su vez, verse afectada la calidad de vida familiar (Rubio, Mena \& Murillo, 2006).

\section{ANTECEDENTES}

La gran mayoría de los estudios que han evaluado la calidad de vida en niños han sido realizados en familias que tienen hijos o hijas con otras discapacidades como síndrome de Down, parálisis cerebral u otras enfermedades intelectuales o enfermedades crónicas degenerativas (Arriola \& Sotelo, 2017, Aya \&
Córdoba, 2013, Benito \& Carpio, 2015, Chávez \& Moncada, 2013, Foley et al. 2014, González, Simón, Cagigal \& Blas, 2013;). Pero pocas investigaciones han valorado la calidad de vida en niños con TDAH, enfocadas en un contexto familiar y, más aún, en el infante en sí mismo (ver tablas 1 y 2) (Barragán \& De la Peña, 2008, Baurmeister, 2014, Elizalde, 2014).

Además, solo se han encontrado dos estudios que han valorado la calidad de vida familiar de niños y niñas con TDAH (Azazy, Nour, Salama \& Ismail, 2018, Córdoba \& Verdugo, 2003) (ver Tabla 2). Se conoce el trastorno que implica el TDAH de manera individual, no obstante, poca atención se le ha dedicado al TDAH en un contexto familiar. Al analizar los estudios de niños y niñas con TDAH en un contexto familiar, por una parte, se ha estudiado la calidad de vida desde una perspectiva de salud mental, relaciones sociales y ambiental (Azazy, Nour, Salama \& Ismail, 2018) y, por otro lado, se ha analizado la calidad de vida familiar con niños con TDAH a través de grupos focales considerando el ambiente médico emocional (Córdoba \& Verdugo, 2003). Sin embargo, no hemos encontrado estudios que hayan investigado la calidad de vida familiar desde una perspectiva multidisciplinar desde las perspectivas del trabajo social y la educación física para enfrentar las implicaciones del TDAH en sus diferentes contextos (médicos, familiar, escolar).

Otro aspecto fundamental que se ha podido analizar tras la revisión de la literatura es que los estudios demuestran que aproximadamente el 80\% de los niños con TDAH son tratados con medicamentos solos o combinados con terapia conductual. Parte del tratamiento farmacológico indicado se encuentra en el grupo de los Ilamados "estimulantes", los cuales han demostrado su efectividad logrando reducir la ansiedad y las conductas repetitivas, favoreciendo el control de la atención; sin embargo, deberá ser el médico tratante quien valore la presencia de efectos secundarios y la pertinencia de continuar con su uso, sin embargo, en muchos de los casos se desconocen si estos tratamientos tendrán efectos adversos a lo largo del tiempo. Además, si un niño con TDAH no es bien tratado en la etapa infantil, 
Quintero-Olivas, D., Romero, E. M., \& Hernández-Murúa, J. A. (2021). Calidad de vida familiar y TDAH infantil. Perspectiva multidisciplinar desde la educación física y el trabajo social. Revista Ciencias de la Actividad Física UCM, No 22(1), enero-junio, 1-17. DOI: http://doi.org/10.29035/rcaf.22.1.1

analizando de una manera integral el contexto familiar e institucional en su etapa adulta, puede presentar altos índices y sintomatológicos, discapacidades, y potencialmente un adulto con problemas de salud mental (Zambrano, Martínez, Río, Dehesa \& Poblano, 2012, Ursano et al., 2018). Debido a esto, se considera pertinente ampliar y analizar las investigaciones desde la disciplina del trabajo social, puesto que, esta nos permitiría un análisis integral de los elementos que rodean la problemática para poder darle una explicación sustancial (Fernández, 2008).

\section{DESARROLLO}

\section{La familia como principal unidad de apoyo para los niños y niñas con TDAH}

Los seres humanos, desde el momento del nacimiento, nos desarrollamos en un contexto social e histórico, siendo la familia el más inmediato. La familia ha sido considerada el núcleo básico de toda sociedad humana, pues, es ahí donde se forman desde su nacimiento todos los individuos. Principalmente, los padres son los encargados de formar individuos comprometidos con su entorno, fomentándoles valores morales que permitirán una convivencia funcional en la sociedad. No obstante, la familia a su vez se encuentra sujeta a influencias mayores y cambiantes desde otros entornos (Papalia, Feldman \& Martorell, 2012, Seelbach, 2012). En la actualidad, existen diferentes tipos de familia; nuclear, monoparental, extensa, entre otras. El papel de la familia como agente socializador, sin duda, es de gran importancia siendo los padres los primeros proveedores de aprendizajes, así como los que establecen normas de comportamiento, valores, actitudes que regirán el comportamiento del grupo y que los encaminarán a la promoción de una calidad de vida familiar. Esta dinámica definirá las redes de apoyo con las que un niño con TDAH pueda contar dentro de su núcleo de referencia, ya que las demandas específicas de esta población requieren de esfuerzos conjuntos (Bello, Rivadeneira, Concha, Soto \& Díaz, 2017).
Lo cierto es que, sin importar como esté conformada la familia, los padres al tener conocimiento que su hijo padece algún trastorno agotan todos los recursos existentes con tal de entender y poder sobrellevar este proceso, buscando dar respuesta a la situación con apoyo de diferentes especialistas (Bauermeister, 2014). Sin duda es un proceso difícil, en el que la familia necesita apoyo y asesoramiento constante durante todo el proceso y su posible canalización a los diferentes especialistas para su tratamiento integral.

\section{Calidad de vida infantil}

El interés por el estudio de la calidad de vida aumentó considerablemente en los últimos años del siglo XX, pues a partir de entonces se consideró que después de haberse satisfecho las necesidades básicas de la población de un mundo desarrollado, era hora de trabajar por mejorar la calidad de vida del individuo. La calidad de vida es considerada como una propiedad que tiene el individuo para experimentar situaciones y condiciones de su ambiente, dependiendo de las interpretaciones y valoraciones que hace de los aspectos objetivos de su entorno (Ardila, 2003).

La calidad de vida ha sido un concepto difícil de definir, por su parte, Nava (2012) considera la calidad de vida como un concepto multidimensional, debido a que éste cambia con las diferentes culturas, épocas y grupos sociales. Además, destaca que la calidad de vida considera que el bienestar está fuertemente ligado a los recursos y comúnmente a la presencia de medios adecuados. Recientemente, se atribuye mayor importancia a la cualidad para obtener satisfacción a través del disfrute de los recursos y medios disponibles, y no sólo de su mera posesión.

Ardila (2003) define que la calidad de vida es un estado de satisfacción general, derivado de la realización de las potencialidades de las personas. Posee aspectos subjetivos y objetivos. Es una sensación subjetiva de bienestar físico, psicológico y social. Incluye como aspectos subjetivos la intimidad, la expresión emocional, la seguridad percibida, la productividad personal y la salud percibida. Como 
Quintero-Olivas, D., Romero, E. M., \& Hernández-Murúa, J. A. (2021). Calidad de vida familiar y TDAH infantil. Perspectiva multidisciplinar desde la educación física y el trabajo social. Revista Ciencias de la Actividad Física UCM, № 22(1), enero-junio, 1-17. DOl: http://doi.org/10.29035/rcaf.22.1.1

aspectos objetivos, el bienestar material, las relaciones armónicas con el ambiente físico, social, con la comunidad y la salud objetivamente percibida. Tal estado de satisfacción es necesario en cada familia para enfrentar los obstáculos que se presenten en el transcurso de su existencia, y tener una vida digna.

En cuanto a su concepción, Arita (2015) dice que la calidad de vida es trabajada científicamente como un constructo muy amplio que abarca diversos significados, pero en lo general, apunta a la búsqueda de la explicación de la buena vida, aquella que se disfruta, que satisface al que la vive y le produce estados de felicidad. El estudio de la calidad de vida debe considerarse desde el paradigma biopsicosocial, pues los aspectos físicos, mentales y sociales son elementos relevantes que influyen en su formación. De acuerdo al Centro sobre Discapacidad de la Universidad de Kansas (2003), por sus siglas en inglés: Beach Center on Disability, proponen cinco aspectos principales para determinar la calidad de vida de las familias: primero, está relacionada con la interacción entren los integrantes de la familia o ambiente familiar; segundo, el papel de los padres hacia los infantes; tercero, el proceso de bienestar emocional entre los integrantes de la familia; cuarto, el apoyo relacionado con los aspectos materiales; y por último, los apoyos necesarios y requeridos para la atención del infante con discapacidad, en nuestro caso, niños y niñas con TDAH.

En la calidad de vida interactúan necesidades a nivel individual y familiar las cuales contemplan aspectos físicos, emocionales y sociales. Sin embargo, su satisfacción depende de los recursos potenciales del individuo y del medio en el cual se desarrolla, en nuestro caso, el contexto familiar (Arita, 2015). Contar con una buena calidad de vida, facilita el afrontamiento de situaciones adversas a las que se puedan enfrentar los individuos. La calidad de vida se ha abordado de manera distinta por diversos autores con diferentes poblaciones y disciplinas. Gifre, Valle, Yuguero, Gil \& Monreal (2010), en un estudio cualitativo con personas adultas con paraplejia y tetraplejia, han abordado la calidad de vida tomando como puntos importantes en un grupo de discusión, las estrategias de afrontamiento, sentimientos, personalización y salud física, para poder analizarla.

En otro estudio, López et al. (2009) han analizado la calidad de vida de cuidadores primarios, en que la han abordado por medio de diferentes escalas; escala de Zarit, perfil de salud de Notting y la escala de ansiedad-depresión de Golberg (EADG-18), que consideran aspectos como: sobrecarga del cuidador, energía, dolor, movilidad, sueño, estado emocional, relaciones sociales, ansiedad y depresión. Estas formas de abordar la calidad de vida han sido de manera individual, desde la disciplina de medicina, rescatando un modelo tradicional biomédico. En relación con la calidad de vida infantil (CVI), Quinceno \& Vinaccia (2013) han analizado diversas formas de abordarla, por mencionar algunas, por medio del cuestionario de salud infantil, por sus siglas en inglés Child Health Questionnaire (CHQ), en la cual se valoran cuestiones como: funciones físicas, rol socialfísico, percepción de la salud, dolor corporal, rol social-comportamiento, autoestima, salud mental, comportamiento general, actividades familiares $y$ cohesión familiar.

Otra forma de abordar la CVI es a través del inventario pediátrico de calidad de vida, por sus siglas en inglés Pediatric Quality of Life (PedsQL), donde se contemplan las siguientes dimensiones: funcionamiento físico, funcionamiento emocional, funcionamiento social y funcionamiento escolar (Quinceno \& Vinaccia, 2013). Por último, en el instrumento de calidad de vida relacionado con la salud para niños y adolescentes (KIDSCREEM) se analizan las dimensiones de bienestar físico y psicológico, autonomía y relación con los padres, amigos, apoyo social y entorno. Estas formas de abordar la calidad de vida infantil abandonan la forma tradicional del modelo biomédico, abordándola desde disciplinas de psicología dando más importancia a las cuestiones psicosociales y a los ambientes en los que se desenvuelve el individuo (Quinceno y Vinaccia, 2013).

Los estudios anteriormente mencionados han abordado la calidad de vida de manera individual, analizando únicamente a la persona que padece alguna enfermedad, sin embargo, desde la disciplina 
Quintero-Olivas, D., Romero, E. M., \& Hernández-Murúa, J. A. (2021). Calidad de vida familiar y TDAH infantil. Perspectiva multidisciplinar desde la educación física y el trabajo social. Revista Ciencias de la Actividad Física UCM, № 22(1), enero-junio, 1-17. DOI: http://doi.org/10.29035/rcaf.22.1.1

de trabajo social será muy pertinente abordar la calidad de vida en relación con la familia y su contexto debido a que la familia y su funcionamiento tiene un papel importante en los procesos de enfermedad, puesto que la esta situación no repercute únicamente en la persona que la padece, sino en la familia en general (Barragán \& De la Peña, 2008, Bauermeister, 2014 y Elizalde, 2014).

Desde la teoría ecológica, se considera que el funcionamiento familiar toma un papel importante y necesario en las familias con niños y niñas con TDAH y será primordial para iniciar un tratamiento integral para el TDAH. De acuerdo con Olson (2011) para el análisis y estudio de la calidad de vida familiar presenta el modelo circunflejo del funcionamiento familiar. En este modelo se distinguen a seis tipos de familias en cuanto a su funcionamiento, la primera de ellas: la familia con cohesión balanceada (para este tipo de familia la unión es prioridad); la segunda, enmarañada (los miembros de esta familia son demasiado dependientes el uno del otro); la tercera, desligada (los miembros de la familia parecen evitar el contacto entre ellos cuando están en casa). La cuarta, con flexibilidad balanceada (en esta familia se puede cambiar el liderazgo); la quinta, rígida (esta familia tiene una regla para casi todas las situaciones posibles); y, por último, la caótica (esta familia nunca se organiza). Las familias que se correspondientes a los tipos enmarañada, desligada, rígida y caótica, es probable que presenten mayores repercusiones a la hora de enfrentar situaciones relacionadas con el TDAH y los posibles tratamientos del mismo (Olson, 2011).

En 1979, David H. Olson, Candy Russel y Douglas Sprenkle, desarrollaron el modelo circunflejo de los sistemas marital y familiar con el objetivo de cubrir la brecha que existía entre la investigación, teoría y práctica. Este modelo integra las dimensiones: cohesión, flexibilidad y comunicación, mismas que se han considerado como relevantes en distintas teorías y abordajes en familia (Costa et al., 2009). Desde este modelo, la dimensión cohesión es definida como los lazos emocionales que los miembros de la familia tienen entre sí: sus indicadores específicos incluyen: cercanía emocional, limites, fronteras, coaliciones, tiempo, espacio, amistades, toma de decisiones, interés y recreación. Desde la dimensión cohesión se pueden identificar cuatro niveles en los grupos familiares: desligadas, separadas conectadas y enmarañadas (Costa et al., 2009). La dimensión flexibilidad es definida como la calidad y expresión del liderazgo, organización, roles, reglas y negociaciones existentes en la familia; sus indicadores específicos incluyen: liderazgo, asertividad, control, disciplina, estilos de negociación, relaciones de rol y reglas relacionadas (Costa et al., 2009). La aplicación de este modelo se considera útil para destacar áreas saludables, (balanceadas) y problemáticas (desbalanceadas) en los sistemas familiares que se estudian. 
Quintero-Olivas, D., Romero, E. M., \& Hernández-Murúa, J. A. (2021). Calidad de vida familiar y TDAH infantil. Perspectiva multidisciplinar desde la educación física y el trabajo social. Revista Ciencias de la Actividad Física UCM, No 22(1), enero-junio, 1-17. DOI: http://doi.org/10.29035/rcaf.22.1.1

Tabla 1

Análisis de la Calidad de Vida de Manera Individual

\begin{tabular}{|c|c|c|}
\hline Autor/es & Muestra & Categorías \\
\hline Urzua (2008) & $\begin{array}{l}240 \text { personas, } 120 \text { de ellas con una } \\
\text { patología médica. }\end{array}$ & $\begin{array}{l}\text { Salud física, salud psicológica, relaciones } \\
\text { sociales y ambiente. }\end{array}$ \\
\hline López et al. (2009) & $\begin{array}{l}156 \text { cuidadores primarios } 156 \text { personas que } \\
\text { no fungen como cuidador primario. }\end{array}$ & $\begin{array}{l}\text { Sobrecarga, energía, dolor, movilidad, } \\
\text { sueño, estado emocional, relaciones } \\
\text { sociales, ansiedad y depresión. }\end{array}$ \\
\hline $\begin{array}{l}\text { Gifre, Valle, Yuguero, Gil \& } \\
\text { Monreal (2010) }\end{array}$ & $\begin{array}{l}18 \text { personas mayores de } 18 \text { años con lesión } \\
\text { medular. }\end{array}$ & $\begin{array}{l}\text { Organización de la vida cotidiana y } \\
\text { limitaciones. }\end{array}$ \\
\hline Arroba (2013) & 60 niños de 5-15 años con discapacidad. & Salud física, rehabilitación y educación. \\
\hline & \multicolumn{2}{|c|}{ Análisis de la Calidad de Vida de Manera Individual-TDAH } \\
\hline $\begin{array}{l}\text { Banaschewski, Soutullo, } \\
\text { Lecendreux, Johnson, } \\
\text { Zuddas, Hodkins, Adeyi \& } \\
\text { Squires (2013) }\end{array}$ & 317 personas de 6-17 años con TDAH. & $\begin{array}{l}\text { Logro, evitación de riesgo, resiliencia, } \\
\text { satisfacción y confort. }\end{array}$ \\
\hline
\end{tabular}

TDAH, Trastorno por déficit de atención e hiperactividad. 
Quintero-Olivas, D., Romero, E. M., \& Hernández-Murúa, J. A. (2021). Calidad de vida familiar y TDAH infantil. Perspectiva multidisciplinar desde la educación física y el trabajo social. Revista Ciencias de la Actividad Física UCM, No 22(1), enero-junio, 1-17. DOI: http://doi.org/10.29035/rcaf.22.1.1

Tabla 2

\section{Análisis de la Calidad de Vida Familiar}

\section{Categorías}

\section{Autor}

Córdoba, Mora, Bedoya \&

Verdugo (2007)

Córdoba, Gómez \& Verdugo

Rodríguez, Verdugo \&

Sánchez (2008)

Fernández, Arias, Gómez \& Jorrín (2012)

Muñoz, Poblete \& Jiménez

53 padres de jóvenes de 16-24 años con discapacidad intelectual.
158 familias con personas mayores de 25 años con discapacidad intelectual.

385 de niños y adolescentes de 3-17 años con discapacidad.

398 padres de 45-65 años con hijos con discapacidad intelectual.
Interacción familiar, rol parental, salud y seguridad, recursos y apoyos.

Interacción familiar, rol parental, salud y seguridad, recursos y a poyos.

Interacción familiar, rol parental, salud y seguridad, recursos y apoyos

51 familiares de personas de 61-93 años con Interacción familiar, rol parental, salud y discapacidad intelectual.

seguridad, recursos y apoyos

Interacción familiar, rol parental, bienestar físico y material, bienestar emocional, funcionamiento familiar y felicidad subjetiva.

Aya \& Córdoba (2013)

18 familias de jóvenes de 16-20 años con discapacidad intelectual.

Interacción familiar, rol parental, salud y seguridad, recursos y apoyos.

González, Simón, Cagigal \& 34 familiares de personas con discapacidad Blas (2013) intelectual.

Interacción familiar, vida familiar diaria, papel de padres y bienestar económico.

Barrera (2015) 25 familias con adolescentes de 10-18 años con hipoacusia. 12 familias con hijos con autismo.

Benito \& Carpio (2015)

Interacción familiar, rol parental, salud y seguridad, recursos y apoyos.

Interacción familiar, rol parental, salud y seguridad, recursos y apoyos, crianza parental y calidad de servicios.
Arriola \& Sotelo (2017)

148 personas con diabetes mellitus tipo 2.

Interacción familiar, rol parental, salud y seguridad, recursos y apoyos y adherencia al tratamiento.

Bello, Rivadeneira, Concha, 316 familias. Interacción familiar, papel de padres, bienestar emocional, bienestar físico y material y recursos y apoyos.

\section{Análisis de la Calidad de Vida Familiar-TDAH}

Córdoba \& Verdugo (2003) 24 familias con niños con TDAH.

Azazy, Nour, Salama \& Ismail 12 familias con niños de 6-14 años con (2018) TDAH.
Bienestar emocional, interacción familiar, bienestar social y bienestar físico.

Salud física, salud psicológica, relaciones sociales, ambiente y funcionamiento familiar.

TDAH, Trastorno por déficit de atención e hiperactividad. 
Quintero-Olivas, D., Romero, E. M., \& Hernández-Murúa, J. A. (2021). Calidad de vida familiar y TDAH infantil. Perspectiva multidisciplinar desde la educación física y el trabajo social. Revista Ciencias de la Actividad Física UCM, № 22(1), enero-junio, 1-17. DOI: http://doi.org/10.29035/rcaf.22.1.1

\section{La calidad de vida de las familias con niños y niñas con TDAH}

En los últimos años el estudio de la calidad de vida familiar en niños con TDAH ha tomado gran importancia en las investigaciones. Córdoba, Gómez \& Verdugo (2008) exponen que hablar de calidad de vida familiar en personas con un trastorno constituye un importante avance en la investigación sobre familia y discapacidad, pues se ha reconocido el importante papel que juega la familia durante el proceso de la enfermedad, discapacidad, las responsabilidades y compromisos que recae en la familia, y, por ende, mismas que afectan su calidad de vida.

Según Llord, Abad, García \& Nieto (1995) la discapacidad o trastorno, en este caso el TDAH puede ser la misma para todas las familias, sin embargo, pueden experimentar el trastorno de diferente manera, dependiendo las circunstancias sociales, la existencia de experiencias tempranas con el TDAH, el grado de fortaleza, creencias y valores, entre otros factores. En algunos casos, las familias no dan importancia a los síntomas que provoca el TDAH en sus niñosy, en otros casos, las familias buscan ayuda rápidamente modificando su vida cotidiana para poder adaptarse al proceso de este trastorno. Por lo general, se puede observar que los padres y madres de niños y niñas con TDAH experimentan diferentes situaciones como: desesperación, sentimientos de culpa, depresión, presión por parte de la sociedad, problemas de comunicación en la familia, problemas relativos a la relación de pareja, problemas en cuanto instituciones médicas o escolares, falta de orientación. En Latinoamérica afecta aproximadamente a 36 millones de personas (Barragán, de la Peña, Ortiz, Ruiz, Hernández, Palacios \& Suárez, 2007, Bauermeister, 2014).

Es importante resaltar el hecho de que, en cualquier familia suele ser un hecho común que se presente alguna vez problema en la interacción del infante con TDAH con uno o más integrantes de la familia, sin embargo, se da el caso en que los niños que tienen un hermano con TDAH, cuya interacción, por las características del niño con trastorno, pudiera presentar una complejidad de mayor nivel causando problemas de conducta e interacción social. Algunas de estas conductas pueden ser: miedo a ser ellos discapacitados, sentimientos de culpa, pena, frustración y excesiva preocupación por el futuro (León, Menés, Puértolas, Trevijano \& Zabalza, 2003, Núñez \& Rodríguez, 2005).

Ante la presencia del TDAH, la situación familiar puede llegar a deteriorarse y la relación entre los padres pasar por momentos difíciles, por no saber cómo dar respuesta a las situaciones que experimentan. Tal situación afecta la calidad de vida familiar. Debería ser esencial que todas las familias cuenten con una buena calidad de vida, con o sin la presencia de un integrante infantil con TDAH. No obstante, es necesario valorar que en familias con niños que presentan TDAH se presentan otras necesidades que pudieran estar fuera del alcance de las familias; tales necesidades no satisfechas se reflejan en la calidad de vida familiar (Rubio, Mena \& Murillo, 2006).

\section{Perspectiva del trabajo social}

Carreón (2014) nos dice que, en virtud de que el trabajo social está inmerso en políticas públicas y programas sociales que van dirigidos a estos grupos vulnerables, es necesario analizar su compromiso considerando su relación con la gestión para el tratamiento de enfermedades y la rehabilitación en instituciones en el sector salud. Entonces, la misión del trabajador trabajo social, consiste en facilitar que todos los individuos logren desarrollar en plenitud sus potencialidades, y a su vez, contribuir para que mejoren su calidad de vida y evitar dificultades que puedan presentarse a lo largo de su vida.

Lorna (2014) define que: "El trabajador social interviene en aquellas dimensiones sociales que determinan las condiciones de vida de la persona, para eliminar o minimizar barreras sociales o para generar, fortalecer o proveer facilitadores sociales para el mismo" (p. 9). El trabajador social interviene en los casos de familias con niños y niñas con TDAH para conocer la calidad de vida y el contexto en el cual se desarrollan las familias, y, basado en los resultados y 
Quintero-Olivas, D., Romero, E. M., \& Hernández-Murúa, J. A. (2021). Calidad de vida familiar y TDAH infantil. Perspectiva multidisciplinar desde la educación física y el trabajo social. Revista Ciencias de la Actividad Física UCM, № 22(1), enero-junio, 1-17. DOI: http://doi.org/10.29035/rcaf.22.1.1

análisis obtenidos, diseñar planes de intervención que aporten a su bienestar.

Tras la revisión temática de la calidad de vida en el infante con TDAH (Tabla 1 y 2), consideramos que, desde la perspectiva del trabajo social, el modelo sistémico aplicado a la intervención profesional y de investigación con familias y otros sistemas, debe tener en cuenta las características de interrelación y de causalidad circular de los componentes entre sí y de estos con el sistema en su totalidad, entendiendo que el todo es más que la suma de las partes. En relación con nuestra profesión, la explicación de un determinado hecho no se encuentra en diferentes causas aisladas, si no en la estructura del sistema y en las relaciones que se dan en él (Fernández, 2008).

El trabajador social actúa para favorecer la canalización e interacción de una persona afectada, en cuanto a la toma de conciencia, movilización de los padres y el grupo familiar, y el entorno social, desde un enfoque de derechos, hacia la resolución de las situaciones que afectan directa o indirectamente el proceso de asimilación, reeducación, rehabilitación y habilitación, de aprendizaje hacia la inclusión social de la persona con algún trastorno, en nuestro caso niños y niñas con TDAH. Destacando que, si un niño con TDAH no se atiende en las etapas infantiles, en el futuro será un adulto potencial para padecer trastornos mentales y esto, a su vez, afectará considerablemente el ambiente familiar (Lorna, 2014).

En casos de TDAH, los obstáculos a los que la familia en general se enfrenta afectan de manera significativa sus ámbitos físicos, psíquicos y sociales, lo que se refleja en su calidad de vida de manera negativa. Por tal motivo, el trabajador social tiene un gran compromiso con esta población; es necesario evaluar si los programas o políticas públicas enfocadas a dichos grupos están diseñados desde un modelo biopsicosocial de salud, de no ser así, elaborar estrategias y propuestas para que sean diseñadas bajo un modelo y un tratamiento integral, ya que la salud se entiende y analiza mejor desde una combinación de factores biológicos, físicos y sociales.

\section{Perspectiva de la educación física}

La investigación reciente indica que los mejores resultados para el tratamiento del niño con TDAH son llevados a cabo de manera individual y con un enfoque multidisciplinario, donde se combine una terapia farmacológica con intervenciones psico-educativas y conductuales. Además, se ha identificado al ejercicio físico como un proceso potencial que al parecer causa modificaciones a nivel estructural y funcional (funciones ejecutivas) en el sistema nervioso central del niño con TDAH. Esto nos lleva a exponer la actividad física como un recurso útil y coadyuvante en el tratamiento de los niños con estas características (Gapin \& Etnier, 2010, Chang, Liu, Yu \& Lee et al., 2012, Benzing \& Schmidt, 2017).

En este sentido, la investigación ha proporcionado evidencias de que el ejercicio físico, en la actualidad, es una alternativa prometedora y una opción para el tratamiento contra el TDAH en nuestros infantes. La literatura científica ha reportado evidencias relativas a los efectos beneficiosos agudos y crónicos, especialmente, el ejercicio cardiovascular con respecto a varias funciones como las cognitivas, conductuales y socio-emocionales (Gapin \& Etnier, 2010, Kang, Choi, Kang \& Han, 2011, Pontifex, Saliba, Raine, Picchietti \& Hillman, 2013, Chang, Hung, Huang, Hatfield \& Hung, 2014). Aunque el ejercicio físico puede representar una opción de tratamiento eficaz en combinación con otros enfoques de tratamiento del TDAH, como la propuesta del presente trabajo desde una perspectiva multidisciplinaria (incluyendo al trabajo social), se necesitan ensayos bien controlados sobre este tema, tanto en niños como en adultos.

\section{Ejercicio físico y TDAH}

Son bien conocidos los efectos positivos del ejercicio físico, incluida la mejora de la forma física (aptitud física), el crecimiento, la densidad mineral ósea y disminución en los niveles de sobrepeso y obesidad, entre otros, en niños normales. Los investigadores en sus estudios han reportado beneficios positivos sobre las funciones psicológicas y cognitivas (Hill, Williams, Aucott, Thomson \& Mon, 2011, Field, 2012, 
Quintero-Olivas, D., Romero, E. M., \& Hernández-Murúa, J. A. (2021). Calidad de vida familiar y TDAH infantil. Perspectiva multidisciplinar desde la educación física y el trabajo social. Revista Ciencias de la Actividad Física UCM, № 22(1), enero-junio, 1-17. DOI: http://doi.org/10.29035/rcaf.22.1.1

Hillman, Erickson \& Kramer, 2008), así como evidencia de los efectos positivos del ejercicio físico sobre las habilidades perceptivas, la inteligencia, el rendimiento académico, el nivel de desarrollo y el rendimiento en pruebas verbales y matemáticas y en las funciones ejecutivas en niños y adolescentes de entre 4 y 18 años (Best, 2010). En este mismo sentido, estudios previos en la literatura científica han demostrado efectos beneficiosos del ejercicio físico en niños con TDAH (Sibley \& Etnier, 2013).

Gapin, Labban \& Etnier (2011) en su revisión concluyeron que el ejercicio físico tiene efectos positivos agudos y crónicos en las medidas conductuales y cognitivas en niños con TDAH, siendo el ejercicio físico un suplemento potencial añadido a la medicación. Archer \& Kostrzewa (2012) reportaron una reducción de los síntomas del TDAH después del ejercicio cardiovascular de intensidad moderada, incluido el control de los impulsos, la falta de atención, el estrés, el afecto negativo (por ejemplo, depresión), la ansiedad y la mala conducta. Otros dos estudios describen una relación entre el ejercicio cardiovascular intenso con la mejora del funcionamiento cognitivo y conductual en niños con TDAH (Halperin \& Healey, 2011, Berwid \& Halperin 2012). En general, se puede determinar que el ejercicio físico se ha establecido como un componente para el tratamiento para niños con TDAH, aunque aún no es clara la idea del impacto sobre su calidad de vida. De acuerdo con la propuesta de esta revisión, es importante entender que el tratamiento del TDAH se tiene que dar desde una perspectiva multidisciplinaria, y así, poder atender diversas áreas de impacto del niño con TDAH; en esta propuesta, la calidad de vida familiar como parte de un entorno primordial donde se desenvuelve el infante. De ahí la idea de que, la atención para el mejoramiento de la calidad de vida debe atenderse desde una perspectiva familiar (aspectos sociales, culturales y económicos, entre otros). Así el tratamiento en otras esferas, como el ejercicio físico, puede impactar secuencialmente con otros tratamientos desde diferentes áreas del conocimiento (desde una perspectiva social).

\section{Educación Física y TDAH}

Aun cuando se ha encontrado sustento científico de que el ejercicio físico es potencial para el tratamiento secuencial para el TDAH, este sigue generando en los padres y la familia, una preocupación por la salud y alteraciones mentales y físicas de sus niños. Otro aspecto de gran interés por parte de los familiares y padres de familia es el rendimiento académico, desenvolvimiento social y rechazo o bullying entre los niños en un ambiente escolar, que en la mayoría de los casos presentan una reacción al tratamiento farmacológico, lo que refuerza la preocupación por los efectos y repercusiones suscitadas a largo plazo (Rief, 2012). Debido a esto, los padres de las familias de niños y niñas con TDAH, consideran que para un mejor desenvolvimiento es necesario un clima favorable de amistad y relaciones interpersonales, llegando así a lograr un estado de ánimo equilibrado con la práctica de la clase de educación física o actividades físicas-deportivas en un ambiente escolar y/o extraescolar (juegos deportivos, otro tipo de actividad organizada). Lo que nos indicaría que la clase de educación física orientada, con objetivos bien definidos, contribuirían a que los niños con TDAH participen de manera placentera, divertida, organizada y supervisada, logrando así un ambiente propicio para su desarrollo no solamente físico, sino con objetivos hacia un desarrollo integrador e integral (Mühlbacher, Rudolph, Lincke \& Nübling, 2009). Existen estudios que han abordado la calidad de vida de una manera individual en niños con TDAH, sin embargo, no se han observado estudios en la literatura científica que hayan abordado la calidad de vida familiar e intervenciones en la clase de educación física de acuerdo con el modelo propuesto por Olso (2011).

\section{PROPUESTA}

Implicaciones de intervención desde una perspectiva del trabajo social y la actividad física en niños y niñas con TDAH

En los últimos años se han buscado múltiples tratamientos para el TDAH desde diferentes 
Quintero-Olivas, D., Romero, E. M., \& Hernández-Murúa, J. A. (2021). Calidad de vida familiar y TDAH infantil. Perspectiva multidisciplinar desde la educación física y el trabajo social. Revista Ciencias de la Actividad Física UCM, № 22(1), enero-junio, 1-17. DOI: http://doi.org/10.29035/rcaf.22.1.1

perspectivas, la médica, psico-educativa y psiquiátrica con el propósito de tener efectos conductuales y estos, a su vez, en la calidad de vida de los infantes con TDAH. Sin embargo, poco se ha escrito o desarrollado sobre la intervención del trabajador social y el educador físico (a través de la actividad física o ejercicio físico) como dos perspectivas profesionales para el desarrollo integral y calidad de vida de los niños con TDAH (Den Heijer et al., 2017, Fernández, 2008, Hernando, 2006).

El trabajo social como profesión se ocupa de promover el cambio social, detectando problemas que se derivan de las relaciones humanas, procurando su solución y fortalecimiento de la sociedad, esto con el fin de crear el bienestar social, mientras que el profesional de la actividad física, desde una perspectiva biológica, procura, a través de toda actividad musculo esquelética, promover un gasto energético y, desde una perspectiva pedagógica, contribuye al desenvolvimiento de los aspectos psico-emocionales y sociales del infante y de interacción social entre ellos. Desde esta perspectiva, hoy en día se debe procurar que las diferentes áreas profesionales interactúen entren sí con objetivos y propósitos comunes (Quintero, 2019).

La propuesta de intervención de la presente revisión teórica hace énfasis en los siguientes puntos: primero, para el mejoramiento de la calidad de vida de los niños con TDAH, no centrado principalmente en la calidad de vida de los niños con TDAH sino en su entorno familiar; segundo, desde una perspectiva de trabajo social, con propósito analizar las condiciones materiales, de salud, culturales y educativas, pero, principalmente la interacción de los aspectos sociales, culturales y de funcionamiento familiar (atención especial) para los niños con TDAH; tercero, desde una perspectiva de la actividad física, promover diferentes programas de actividad física-deportiva y/o educación física como una actividad lúdica educativa en un contexto educativo (clases de educación física en la educación básica) y en diferentes contextos educativos no formales (por ejemplo, actividad deportiva, programas de acondicionamiento físico), como medios de desenvolvimiento del infante para modular la interacción conductual intra e interpersonal (Carreón, 2014, Quintero, 2019). De esta manera, se procura evitar tratamientos psiquiátricos, médicos y farmacológicos que, en su mayoría, hacen del infante un farmacodependiente; por último, para el tratamiento integral del niño con TDAH se requiere considerar la teoría ecológica, que pondera como papel fundamental el funcionamiento familiar para la mejora de la calidad de vida de la familia del infantey, no necesariamente, la propia calidad de vida del infante y además, tomar como referencia el modelo circunflejo del funcionamiento familiar propuesto por Olso (2011).

\section{CONCLUSIONES}

La educación física y el trabajo social son dos áreas de desarrollo profesional que pueden aportar evidencias sustanciales para el desarrollo integrador, integral y calidad de vida familiar en los niños y niñas con TDAH. El estudio de la calidad de vida en niños y niñas con TDAH no solo se debe abordar desde una perspectiva individual sino teniendo claro el ambiente familiar y su desenvolvimiento para el tratamiento integral de niños y niñas con TDAH. La teoría ecológica y el modelo circunflejo de Olso abordado desde una perspectiva del trabajo social podrían apoyarnos para un abordaje teórico para el tratamiento integral del TDAH. La clase de educación física puede ser un escenario organizado y supervisado donde se pueden desarrollar muchas habilidades integradoras en niños y niñas con TDAH. Existen un conjunto de evidencias científicas con respecto a los efectos del ejercicio físico en las áreas cognitivas, conductuales, socioemocionales y (neuro) fisiológicos en el TDAH. El ejercicio físico representa una alternativa prometedora o una opción de tratamiento adicional para niños y niñas con TDAH. 
Quintero-Olivas, D., Romero, E. M., \& Hernández-Murúa, J. A. (2021). Calidad de vida familiar y TDAH infantil. Perspectiva multidisciplinar desde la educación física y el trabajo social. Revista Ciencias de la Actividad Física UCM, No 22(1), enero-junio, 1-17. DOI: http://doi.org/10.29035/rcaf.22.1.1

\section{REFERENCIAS BIBLIOGRÁFICAS}

Archer, T., \& Kostrzewa, R. M. (2012). Physical exercise alleviates ADHD symptoms: regional deficits and development trajectory. Neurotoxicity research, 27(2), 195-209. DOI: https://doi.org/10.1007/s12640-011-9260-0

Ardila, R. (2003). Calidad de vida: una definición integradora. Revista Latinoamericana de Psicología, 35(2.), 161-164. Recuperado de https://www.redalyc.org/articulo.oa?id=80535203

Arita, B. Y. (2015). Modelo sistémico de percepción de la calidad de vida. México: Editorial Universitaria.

Arriola, C. E., \& Sotelo, C. J. (2017). Influencia de la calidad de vida familiar en la adherencia al tratamiento farmacológico de la diabetes mellitus tipo 2 de los pacientes que acuden al club dulce vida del centro de salud la concordia. (Tesis de especialidad). Pontificia Universidad Católica del Ecuador, Concordia, Ecuador. Recuperado de http://reposito-

rio.puce.edu.ec/handle/22000/12862?show =full

Arroba, N. G. (2013). Diagnóstico de los tipos de discapacidad y calidad de vida de niñas, niños y adolescentes, atendidos por la congregación de La Providencia en Quito (Tesis de especialización). PUCE, Quito, Ecuador. Recuperado de http://repositorio.puce.edu.ec/handle/22000/6058

Aya, V., \& Córdoba, L. (2013). Asumiendo juntos los retos: calidad de vida en familias de jóvenes con discapacidad intelectual. Revista de la Facultad de Medicina. 67(2.), 155-166. Recuperado de https://revistas.unal.edu.co/index.php/revfacmed/article/view/40169
Azazy, S., Nour-Eldein, H., Salama, H., \& Ismail, M. (2018). Quality of life and family function of parents of children with attention deficit hyperactivity disorder. East Mediterranean Health Journal, 24(6), 579-587. DOI: https://doi.org/10.26719/2018.24.6.579

Banaschewski, T., Soutullo, C., Lecendreux, M., Johnson, M., Zuddas, A., Hodkins, P., Adeyi, B., \& Squires, L. A. (2013). Health-related quality of life and functional outcomes from a randomized, controlled study of lisdexamfetamine dimesylate in children and adolescents with attention deficit hyperactivity disorder. CNS Drugs, 27(10), 829-840. DOI: https://doi.org/10.1007/s40263-013-0095-5

Barragán, E., \& de la Peña, F. (2008). Primer consenso latinoamericano y declaración de México para el trastorno de déficit de atención e hiperactividad en Latinoamérica. Revista Médica Hondureña, 76(1), 33-38. Recuperado de http://repositorio.inprf.gob.mx/handle/123456789/5282

Barragán-Pérez, E., de la Peña-Olvera, F., Ortiz-León, S., Ruiz-García, M., Hernández-Aguilar, J., Palacios-Cruz, L., \& Suárez-Reynaga, A. (2007). Primer consenso latinoamericano de trastorno por déficit de atención e hiperactividad. Boletín Médico del Hospital Infantil de México, 64(5), 326-343. Recuperado de https://www.medigraphic.com/cgi-bin/new/resumen.cgi?|DARTICULO=13657

Barrera, L. (2015). Calidad de vida familiar en los adolescentes de 10 a 18 años con hipoacusia del Instituto Nacional de Audición y Lenguaje en la ciudad de Quito, en el periodo de enero a mayo 2015 (Tesis de especialidad). Pontificia Universidad Católica del Ecuador, Quito, Ecuador.

Bauermeister, J. J. (2014). Hiperactivo, impulsivo, distraído ¿Me conoces?: Guía Acerca del Déficit Atencional (TDAH) Para Padres, Maestros y Profesionales. New York: The Guilford press. 
Quintero-Olivas, D., Romero, E. M., \& Hernández-Murúa, J. A. (2021). Calidad de vida familiar y TDAH infantil. Perspectiva multidisciplinar desde la educación física y el trabajo social. Revista Ciencias de la Actividad Física UCM, No 22(1), enero-junio, 1-17. DOI: http://doi.org/10.29035/rcaf.22.1.1

Beach Center on Disability. (2003). Families. Recuperado de https://beachcenter.Isi.ku.edu/beach-families

Bello-Escamilla, N., Rivadeneira, J., Concha-Toro, M., Soto-Caro, A y Díaz-Martínez, X. (2017). Escala de Calidad de Vida Familiar: validación y análisis en población chilena. Universitas Psychologica, 16(4), 1-10. DOI: https://doi.org/10.11144/Javeriana.upsy164.ecvf

Benito, E., \& Carpio, M. (2015). Métodos de evaluación familiar: escolares con trastornos del espectro autista. Opción, 37(2), 122-143. Recuperado de https://www.redalyc.org/pdf/310/31045568009.pdf

Benzing, V., \& Schmidt, M. (2017). Cognitively and physically demanding exergaming to improve executive functions of children with attention deficit hyperactivity disorder: a randomised clinical trial. BMC pediatrics, 17, 1-8. DOI: https://doi.org/10.1186/s12887016-0757-9

Berwid, O. G., \& Halperin, J. M. (2012). Emerging support for a role of exercise in attention-deficit/hyperactivity disorder intervention planning. Current psychiatry reports, 74(5), 543-551. DOl: https://doi.org/10.1007/s11920$012-0297-4$

Best, J. R. (2010). Effects of physical activity on children's executive function: Contributions of experimental research on aerobic exercise. Developmental Review, 30(4), 331-351. DOI: https://doi.org/10.1016/j.dr.2010.08.001

Carreón, J. (2014). Compromiso laboral del trabajo social en el sector salud. Contaduría y Administración, 60(1), 31-51. DOI: http://dx.doi.org/10.1016/S01861042(15)72146-7
Chang, Y. K., Hung, C. L., Huang, C. J., Hatfield, B. D., \& Hung, T. M. (2014). Effects of an aquatic exercise program on inhibitory control in children with ADHD: a preliminary study. Archives of Clinical Neuropsychology, 29(3), 217-223. DOl: https://doi.org/10.1093/arclin/acu003

Chang, Y. K., Liu, S., Yu, H. H., \& Lee, Y. H. (2012). Effect of acute exercise on executive function in children with attention deficit hyperactivity disorder. Archives of Clinical Neuropsychology, 27(2), 225-237. DOl: https://doi.org/10.1093/arclin/acr094

Chávez, M., \& Moncada, K. (2013). Calidad de vida familiar en padres de personas con discapacidad intelectual. (Tesis de pregrado). Universidad Rafael Urdaneta, Maracaibo, Venezuela.

Córdoba, L., \& Verdugo, M, A. (2003). Aproximación a la calidad de vida de familias de niños y niñas con TDAH un enfoque cualitativo, Siglo Cero, Revista Española sobre Discapacidad Intelectual, 34(4), 19-33. Recuperado de

http://riberdis.cedd.net/handle/11781/3075

Córdoba, L., Mora, A., Bedoya, A., \& Verdugo, M. (2007). Familias de adultos con discapacidad intelectual en Cali, Colombia, desde el modelo de calidad de vida. Psykhe, 16(2), 29-42. DOI: http://dx.doi.org/10.4067/s071822282007000200003

Córdoba, L., Gómez, J., \& Verdugo, M. (2008). Calidad de vida familiar en personas con discapacidad: un análisis comparativo. Universitas Psychologica, 7(2), 369-383. Recuperado de https://revistas.javeriana.edu.co/index.php/revPsycho/article/view/369 
Quintero-Olivas, D., Romero, E. M., \& Hernández-Murúa, J. A. (2021). Calidad de vida familiar y TDAH infantil. Perspectiva multidisciplinar desde la educación física y el trabajo social. Revista Ciencias de la Actividad Física UCM, No 22(1), enero-junio, 1-17. DOI: http://doi.org/10.29035/rcaf.22.1.1

Costa, D., Gonzalez, M., Masjuan, N., Trápaga, M, A., Del arca, D., Scafarelli, L. \& Feibuscheurez, A. (2009). Escala de evaluación del funcionamiento familiar-FACES I- proceso de adaptación a Montevideo, Uruguay. Ciencias Psicológicas, 3(11), 43-56. Recuperado de http://www.scielo.edu.uy/scielo.php?script =sci_arttext\&pid=S1688-

42212009000100005

Den Heijer, A. E., Groen, Y., Tucha, L., Fuermaier, A. B., Koerts, J., Lange, K. W., ... \& Tucha, O. (2017). Sweat it out? The effects of physical exercise on cognition and behavior in children and adults with ADHD: a systematic literature review. Journal of Neural Transmission, 124(1), 3-26. DOI: https://doi.org/10.1007/s00702-016-1593-7

Elizalde, A. (2014). TDAH: repercusión en la vida del niño. Diseño de una intervención de enfermería en el programa de atención al niño sano. (Tesis de pregrado). Universidad Pública de Navarra, Navarra, España. Recuperado de https://academica-e.unavarra.es/handle/2454/11552

Fernández, T. (2008). Trabajo social con casos. España: Alianza.

Fernández-Faúndez, E, M., Arias, B., Gómez, L, E., \& Jorrín, I, M. (2012). Calidad de vida en familias de personas mayores con discapacidad intelectual. Siglo Cero, Revista Española sobre Discapacidad Intelectual, 43(4), 3148. DOI: https://doi.org/10.11144/Javeriana.upsy76-4.ecvf

Field, T. (2012). Exercise research on children and adolescents. Complementary Therapies in Clinical Practice, 18(1), 54-59. DOI: https://doi.org/10.1016/j.ctcp.2011.04.002
Foley, K, R., Girdler, J., Dows, P., Jacoby, J., Bourke, N., Einfeld, G..., Leonard, H. (2014). Relación entre la calidad de vida familiar y las ocupaciones diarias de jóvenes con síndrome de Down. Revista Sindrome de Down, 37, 196203. Recuperado de http://revistadown.downcantabria.com/wp-content/uploads/2014/12/revista123_196203.pdf

Gapin, J. I., Labban, J. D., \& Etnier, J. L. (2011). The effects of physical activity on attention deficit hyperactivity disorder symptoms: The evidence. Preventive Medicine, (52), S70-S74. DOI: https://doi.org/10.1016/j.ypmed.2011.01.022

Gapin, J., \& Etnier, J. L. (2010). The relationship between physical activity and executive function performance in children with attention-deficit hyperactivity disorder. Journal of Sport and Exercise Psychology, 32(6), 753-763.

DOI: https://doi.org/10.1123/jsep.32.6.753

Gifre, M., Valle, A., Yuguero, M., Gil, Á., \& Monreal, P. (2010). La mejora de la calidad de vida de las personas con lesión medular: La transición del centro rehabilitador a la vida cotidiana desde la perspectiva de los usuarios. Athenea Digital. Revista de Pensamiento e Investigación Social, (18), 3-15. DOI: https://doi.org/10.5565/rev/athenead/v0n18.708

González del Yerro, A., Simón Rueda, C., Cagigal Gregorio, V., \& Blas Gómez, E. (2013). La calidad de vida de las familias de personas con discapacidad intelectual. Un estudio cualitativo realizado en la comunidad de Madrid. Reop, 24(7.), 93-109. DOI: https://doi.org/10.5944/reop.vol.24.num.1.20 13.11273 
Quintero-Olivas, D., Romero, E. M., \& Hernández-Murúa, J. A. (2021). Calidad de vida familiar y TDAH infantil. Perspectiva multidisciplinar desde la educación física y el trabajo social. Revista Ciencias de la Actividad Física UCM, No 22(1), enero-junio, 1-17. DOI: http://doi.org/10.29035/rcaf.22.1.1

Halperin, J. M., \& Healey, D. M. (2011). The influences of environmental enrichment, cognitive enhancement, and physical exercise on brain development: Can we alter the developmental trajectory of ADHD? Neuroscience \& Biobehavioral Reviews, 35(3), 621634. DOI: https://doi.org/10.1016/j.neubiorev.2010.07.006

Hernando Sanz, M.A. (2006). Calidad de vida, educación física y salud. Revista española de pedagogía, 64(235), 453-464. Recuperado de https://revistadepedagogia.org//xiv/no235/calidad-de-vida-educacion-fisica-y-salud/101400009870/

Hill, L. J., Williams, J. H., Aucott, L., Thomson, J., \& Mon-Williams, M. A. R. K. (2011). How does exercise benefit performance on cognitive tests in primary-school pupils? Developmental Medicine \& Child Neurology, 53(7), 630-635. DOI: https://doi.org/10.1111/j.14698749.2011.03954.x

Hillman, C. H., Erickson, K. I., \& Kramer, A. F. (2008). Be smart, exercise your heart: exercise effects on brain and cognition. Nature reviews neuroscience, (9), 58-65. DOl: https://doi.org/10.1038/hrn2298

Kang, K. D., Choi, J. W., Kang, S. G., \& Han, D. H. (2011). Sports therapy for attention, cognitions and sociality. International Journal of Sports Medicine, 32(12), 953-959. Recuperado de https://www.thieme-connect.de/products/ejournals/abstract/10.1055/s-0031-1283175

León, R., Menés, M. M., Puértolas, N., Trevijano, I., \& Zabalza, S. (2003). El impacto en la familia del deficiente mental. Revista Interuniversitaria de Formación de Profesorado, 17(2), 195-212. Recuperado de https://www.redalyc.org/pdf/274/27417211.pdf

Llord, A., Abad, M. A., García, M., \& Nieto, J. (1995). Ciencias psicosociales, aplicadas a la salud. México: Interamericana Mc Graw Hill.
López Gil, M. J., Orueta Sánchez, R., Gómez-Caro, S., Sánchez Oropesa, A., Carmona de la Morena, J., \& Alonso Moreno, F. J. (2009). El rol del cuidador de personas dependientes y sus repercusiones sobre la calidad de vida y su salud. Revista Clínica de Medicina Familiar, 2(7), 332-334. Recuperado de http://scielo.is-

ciii.es/scielo.php?script=sci_arttext\&pid=S1 699-695X2009000200004

Lorna, S. (2014). El Trabajo Social en el área de discapacidad: especificidades del rol profesional. XXVII Congreso Nacional de Trabajo Social. Congreso llevado a cabo en Paraná, Argentina. Recuperado de https://issuu.com/faapss/docs/sidebottom_lorna

Mühlbacher, A. C., Rudolph, I., Lincke, H. J., \& Nübling, M. (2009). Preferences for treatment of attention-deficit/hyperactivity disorder (ADHD): a discrete choice experiment. BMC health services research, 9, 149. DOI: https://doi.org/10.1186/1472-6963-9-149

Muñoz, Y. A., Poblete Y. P., \& Jiménez, E. (2012). Calidad de vida familiar y bienestar subjetivo en jóvenes con discapacidad intelectual de un establecimiento con educación especial y laboral de la ciudad de Talca. Interdisciplinaria, 29(2), 207-221. Recuperado de https://www.redalyc.org/articulo.oa?id=18026361003

Nava, M. G. (2012). La calidad de vida: Análisis multidimensional. Enfermería Neurológica, 77(3), 129-137. Recuperado de https://www.medigraphic.com/cgi-bin/new/resumen.cgi?IDARTICULO=38334

Núñez, B., \& Rodríguez, L. (2005). Los hermanos de personas con discapacidad: una asignatura pendiente. Buenos Aires: Asociación AMAR.

Olson, D. (2011). FACES IV and the cirmplex model: validatios study. Journal of Marital and Family Therapy, 37(1), 64-80. DOI: https://doi.org/10.1117/j.1752 0606.2009.00175.x 
Quintero-Olivas, D., Romero, E. M., \& Hernández-Murúa, J. A. (2021). Calidad de vida familiar y TDAH infantil. Perspectiva multidisciplinar desde la educación física y el trabajo social. Revista Ciencias de la Actividad Física UCM, No 22(1), enero-junio, 1-17. DOI: http://doi.org/10.29035/rcaf.22.1.1

Papalia, D. E., Feldman, R.D., \& Martorell, G. (2012). Desarrollo humano. México: Mc Graw Hill.

Pontifex, M. B., Saliba, B. J., Raine, L. B., Picchietti, D. L., \& Hillman, C. H. (2013). Exercise improves behavioral, neurocognitive, and scholastic performance in children with attentiondeficit/hyperactivity disorder. The Journal of pediatrics, 162(3), 543-551. DOI: https://doi.org/10.1016/j.jpeds.2012.08.036

Quiceno, J. M., \& Vinaccia, S. (2013). Calidad de vida relacionada con la salud infantil: una aproximación desde la enfermedad crónica. Psychologia: Avances de la Disciplina, 7(2), 69-86. Recuperado de http://www.scielo.org.co/pdf/psych/v7n2/v7 n2a06.pdf

Quintero, J., \& Castaño de la Mota, C. (2014). Introducción y etiopatogenia del trastorno por déficit de atención e hiperactividad (TDAH). Pediatría Integral, 18(9), 600-608. Recuperado de https://www.pediatriaintegral.es/wp-con-

tent/uploads/2014/xviii09/02/n9-600-

608_Javier\%20Quintero.pdf

Quintero-Olivas, DK. (2019). Calidad de vida de familias con niños y niñas con trastorno por deficit de atención con hiperactividad (TDAH) atendidos en el laboratorio de psicomotricidad de la FEFYDE-UAS (Tesis de maestría). Universidad Autónoma de SinaIoa, Culiacán, México. Recuperado de http://fts.uas.edu.mx/wp-content/uploads/2020/O7/DIANA-K.-QUINTERO-O.-2019.pdf

Rief, S. F. (2012). How to reach and teach children with ADD/ADHD: Practical techniques, strategies, and interventions (Vol. 3). John Wiley \& Sons.
Rodríguez, A., Verdugo, M, A., \& Sánchez, M., (2008). Calidad de vida familiar y apoyos para los progenitores de personas con discapacidad intelectual en proceso de envejecimiento. Siglo Cero. Revista Española sobre Discapacidad Intelectual, 39(3), 19-34. Recuperado de https://sid.usal.es/idocs/F8/ART0929/calidad_vida_familiar.pdf

Rubio. L. Mena, B., \& Murillo, B. (2006). El pediatra y la familia de un niño con TDAH. Revista de Pediatría en Atención Primaria, 8(Suple.4), 199-216. Recuperado de https://www.fundacionadana.org/wp-content/uploads/2017/01/el-pediatra-y-la-familia-de-un-nio-con-tdah.pdf

Secretaria de Salud (SSA). (2002). Programa específico de trastorno por déficit de atención 2001-2006. México. Recuperado de http://www.salud.gob.mx/unidades/cdi/documentos/pasm_intro.pdf

Seelbach, G, A. (2012). Problemas psicosociales en México. México: Red Tercer Milenio.

Sibley, B. A., \& Etnier, J. L. (2003). The relationship between physical activity and cognition in children: a meta-analysis. Pediatric exercise science, 15(3), 243-256. DOI: https://doi.org/10.1123/pes.15.3.243

Ursano, R. J., Kessler, R. C., Naifeh, J. A., Mash, H. B. H., Nock, M. K., Aliaga, P. A., ... \& Sampson, N. A. (2018). Risk factors associated with attempted suicide among US army soldiers without a history of mental health diagnosis. JAMA Psychiatry, 75(10), 1022-1032. DOI: https://doi.org/10.1001/jamapsychiatry.2018.2069

Zambrano-Sánchez, E., Martínez-Cortés, J. A., RíoCarlos, Y. D., Dehesa-Moreno, M., \& Poblano, A. (2012). Low quality of life scores in school children with attention deficit-hyperactivity disorder related to anxiety. Arquivos de neuro-psiquiatria, 70(3), 180-184. DOI: https://doi.org/10.1590/s0004282X2012000300005 
Quintero-Olivas, D., Romero, E. M., \& Hernández-Murúa, J. A. (2021). Calidad de vida familiar y TDAH infantil. Perspectiva multidisciplinar desde la educación física y el trabajo social. Revista Ciencias de la Actividad Física UCM, No 22(1), enero-junio, 1-17. DOl: http://doi.org/10.29035/rcaf.22.1.1

\section{Dirección para correspondencia}

Dr. José Aldo Hernández-Murúa

Doctor en Ciencias.

Facultad de Educación Física y Deporte,

Universidad Autónoma de Sinaloa.

Culiacán, México.

Dirección postal: Calle Universitarios y Calz de las Americas Norte s/n

Cd. Universitaria, 80013 Culiacán Rosales, Sin.

Culiacán, México

ORCID: https://orcid.org/0000-0002-9277-5799

Contacto:

aldohdez80@hotmail.com

Recibido: 14-04-2020

Aceptado: 27-08-2020 